\title{
THE BAIRE ORDER OF THE FUNCTIONS CONTINUOUS ALMOST EVERYWHERE
}

\author{
R. D. MAULDIN
}

\begin{abstract}
Let $\Phi$ be the family of all real-valued functions defined on the unit interval $I$ which are continuous except for a set of Lebesgue measure zero. Let $\Phi_{0}$ be $\Phi$ and for each ordinal $\alpha$, let $\Phi_{\alpha}$ be the family of all pointwise limits of sequences taken from $\bigcup_{\gamma<\alpha} \Phi_{\gamma}$. Then $\Phi_{\omega_{1}}$ is the Baire family generated by $\Phi$. It is proven here that if $0<\alpha<\omega_{1}$, then $\Phi_{\alpha} \neq \Phi_{\omega_{1}}$. The proof is based upon the construction of a Borel measurable function $h$ from $I$ onto the Hilbert cube $Q$ such that if $x$ is in $Q$, then $h^{-1}(x)$ is not a subset of an $F_{\sigma}$ set of Lebesgue measure zero.
\end{abstract}

If $\Phi$ is a family of real-valued functions defined on a set $S$, then the Baire family generated by $\Phi$ may be described as follows: Let $\Phi_{0}=\Phi$ and for each ordinal $\alpha>0$, let $\Phi_{\alpha}$ be the family of all pointwise limits of sequences taken from $\bigcup_{\gamma<\alpha} \Phi_{\gamma}$. Of course, $\Phi_{\omega_{1}}=\Phi_{\omega_{1}+1}$, where $\omega_{1}$ denotes the first uncountable ordinal and $\Phi_{\omega_{1}}$ is the Baire family generated by $\Phi$; the family $\Phi_{\omega_{1}}$ is the smallest subfamily of $R^{S}$ containing $\Phi$ and which is closed under pointwise limits of sequences. The order of $\Phi$ is the first ordinal $\alpha$ such that $\Phi_{\alpha}=\Phi_{\alpha+1}$.

Let $C$ denote the family of all real-valued continuous functions on the unit interval $I$. It was first proven by Lebesgue that the order of $C$ is $\omega_{1}$ [1]. In 1924, Kuratowski [2] proved that if one relaxes the continuity condition by only requiring that the original functions be continuous except for a first category set, then the Baire order of this enlarged family is 1. In 1930, Kantorovitch [3] showed that if one requires that the original functions be continuous except for a set of Lebesgue measure zero, then the Baire order of this family is at least 2 . Recently, the author generalized this result in the following fashion [4].

THEOREM. Let $S$ be a complete separable metric space, let $u$ be a $\sigma$-finite, complete Borel measure on $S$ and let $\Phi$ be the family of all realvalued functions on $S$, whose set of points of discontinuity is of u-measure 0 . Then (1) the order of $\Phi$ is 1 if and only if $u$ is a purely atomic measure whose

Received by the editors December 16, 1972.

AMS (MOS) subject classifications (1970). Primary 28A05, 26A21; Secondary 04A15, $54 \mathrm{C} 50$.

Key words and phrases. Lebesgue measure zero, Baire class $\alpha$, universal function, Hilbert cube.

(c) American Mathematical Society 1973 
set of atoms is dispersed and (2) if the order of $\Phi$ is not 1 , the order of $\Phi$ is at least 3.

In this paper $\Phi$ will denote the family of all real-valued functions defined on the unit interval $I$ which are continuous except for a set of Lebesgue measure zero. It is shown here that the Baire order of this family is $\omega_{1}$. The method of proof involves showing that there is a Borel measurable function $h$ from $I$ onto the Hilbert cube such that if $x$ is a point of the Hilbert cube, then $h^{-1}(x)$ is not a subset of an $F_{\sigma}$ set of Lebesgue measure 0 . Of course, there is no such function $h$ which is continuous or even an $h$ such that $h^{-1}(x)$ is an $F_{\sigma}$ set for each $x$. Thus, the function $h$ is necessarily fairly complicated. We begin with a sequence of lemmas which are used to demonstrate the existence of one such function $h$. This function will be used to construct a transfinite sequence of "universal functions" $\left\{U_{\alpha}\right\}_{0<\alpha<\omega_{1}}$ [Theorem 2]. Finally, a diagonal type argument is applied to prove that the order of $\Phi$ is $\omega_{1}$ [Theorem 4].

LEMMA 1. Let $P$ be a perfect subset of the interval I such that if an open set $U$ meets $P$, then $\lambda(P \cap U)>0$. There is a double sequence $\left\{F_{n p}\right\}_{n, p=1}^{\infty}$ of disjoint perfect subsets of $P$ such that (1) each $F_{n p}$ is nowhere dense in $P$ and if an open set $U$ meets $F_{n p}$, then $\lambda\left(U \cap F_{n p}\right)>0$, and (2) if $n$ is a positive integer and $U$ is a nonempty set open with respect to $P$, then there is some $p$ such that $F_{n p}$ is a subset of $U$.

Proof. Let $\left\{s_{n}\right\}_{n=1}^{\infty}$ be a countable base of nonempty open sets with respect to $P$.

Let $K_{11}$ be a perfect set lying in $s_{1} \cap s_{1}=s_{1}$ such that $K_{11}$ is nowhere dense in $P$ and if an open set $U$ intersects $K_{11}$, then $\lambda\left(K_{11} \cap U\right)>0$. For each positive integer $n$ and integer $p, 1 \leqq p \leqq n+1$, let $K_{n+1}{ }_{p}$ be $\varnothing$, if $s_{n+1} \cap s_{p}=\varnothing$, and, if $s_{n+1} \cap s_{p} \neq \varnothing$ let $K_{n+1 \nu}$ be a perfect set lying in $s_{n+1} \cap s_{p}$ such that (1) $K_{n+1}$ is nowhere dense in $P$, (2) $K_{n+1 p}$ is disjoint from $\left(\bigcup_{r=1}^{n} \bigcup_{q=1}^{r} K_{r q}\right) \cup\left(\bigcup_{i=1}^{p-1} K_{n+1} i\right)$ (a union from 1 to 0 is taken to be empty) and (3) if an open set $U$ intersects $K_{n+1}$, then $\lambda\left(K_{n+1} \cap U\right)>0$.

For each $p$, let $F_{1 p}=K_{p p}$. For each positive integer pair $n, p$, let $F_{n+1} p$ be the first term of the sequence $\left\{K_{q p}\right\}_{q=p}^{\infty}$ which follows $F_{n p}$ and which is nonempty. ties.

It follows that the double sequence $\left\{F_{n, p}\right\}_{p, n=1}^{\infty}$ has the required proper-

Now let $\left\{F_{(n, p)}\right\}_{n, p=1}^{\infty}$ be a double sequence which has the properties listed in Lemma 1, where $P$ is the interval $[0,1]$.

By repeated application of Lemma 1, we have

LeMma 2. There is a system of sets $\left\{F_{\left(n_{1}, n_{2}, \cdots, n_{2 k}\right)}\right\}$, where $\left(n_{1}, \cdots, n_{2 k}\right)$ ranges over the family of all finite sequences of positive integers of even 
length such that if $\left(n_{1}, n_{2}, \cdots, n_{2 k-1}, n_{2 k}\right)$ is such a sequence, then the double sequence $\left\{F_{\left(n_{1}, n_{2}, \cdots, n_{2 k-1}, n_{2 k}, n, p\right)}\right\}_{n, p=1}^{\infty}$ has the properties listed in Lemma 1 with respect to the set $\left\{F_{\left(n_{1}, n_{2}, \cdots, n_{2 k-1}, n_{2 k}\right)}\right\}$.

Let $W_{n}$ be the family $\left\{F_{(n, p)}\right\}_{p=1}^{\infty}$ for each $n$, and for each finite sequence of positive integers $\left(n_{1}, \cdots, n_{k}\right)$, let $W_{\left(n_{1}, \cdots, n_{k}\right)}$ be the family

$$
\left\{F_{\left(n_{1}, i_{1}, n_{2}, i_{2}, \cdots, n_{k}, i_{k}\right)}\right\}
$$

where $\left(i_{1}, \cdots, i_{k}\right)$ ranges over all $k$-tuples of positive integers. Let $T_{n_{1}, \cdots, n_{k}}$ be the union of all the sets in the family $W_{\left(n_{1}, \cdots, n_{k}\right)}$.

Notice that these families have the following three properties:

(1) If $\left(m_{1}, \cdots, m_{k}\right) \neq\left(n_{1}, \cdots, n_{k}\right)$, then $T_{\left(n_{1}, \cdots, n_{k}\right)}$ and $T_{\left(m_{1}, \cdots, m_{k}\right)}$ are disjoint;

(2) Each set in $W_{\left(n_{1}, \cdots, n_{k}, n_{k+1}\right)}$ is a subset of some set in $W_{\left(n_{1}, \ldots, n_{k}\right)}$; and

(3) If $F \in W_{\left(n_{1}, \cdots, n_{k}\right)}, n$ is a positive integer, and $U$ is an open set which meets $F$, then there is some set in the family $W_{\left(n_{1}, \cdots, n_{k}, n\right)}$ which is a subset of $U$.

LEMMA 3. Let $\left\{n_{k}\right\}_{k=1}^{\infty}$ be a sequence of positive integers. The intersection of the monotonically decreasing sequence $\left\{T_{\left(n_{1}, \cdots, n_{k}\right)}\right\}_{k=1}^{\infty}$ is not a subset of an $F_{\sigma}$ set of measure 0.

Proof. For each $n$, let $A_{n}$ be a closed set of Lebesgue measure 0 . Since $T_{n_{1}}$ is dense in the interval $I$, it follows that there is some set $F_{n_{1}, k_{1}}$ which does not intersect $A_{1}$.

Since $\lambda\left(F_{\left(n_{1}, k_{1}\right)}\right)>0$ and $\lambda\left(A_{2}\right)=0$, there is an open set which meets $F_{n_{1}, k_{1}}$ which does not intersect $A_{2}$. It follows from property (3) that there is a set $F_{\left(n_{1}, k_{1}, n_{2}, k_{2}\right)}$ which is a subset of $F_{\left(n_{1}, k_{1}\right)}$ and does not meet $A_{2}$.

Continuing this process, we obtain a monotonically decreasing sequence $\left\{F_{\left(n_{1}, k_{1}, \cdots, n_{p}, k_{p}\right)}\right\}_{p=1}^{\infty}$ such that for each $p, F_{\left(n_{1}, k_{1}, \cdots, n_{p}, k_{p}\right)}$ does not intersect $A_{\boldsymbol{p}}$. The nonempty intersection of this sequence of sets is a subset of $\bigcap_{k=j}^{\infty} T_{\left(n_{1}, \cdots, n_{k}\right)}$ which does not intersect $\bigcup_{n=1} A_{n}$. This completes the proof of Lemma 3.

For each $k$, let $H_{k}=\bigcup T_{n_{1}, \ldots, n_{k}}$, where the union is taken over all $k$-tuples of positive integers. Let $H=\bigcap_{k=1}^{\infty} H_{k}$. The set $H$ is an $F_{\sigma \delta}$ set.

Let $\mathscr{N}$ denote the space of all irrational numbers between 0 and 1 . Identify the space of all infinite sequences of positive integers with the space via the continued fraction expansion of the members of the space $\mathscr{N}$. If $Z \in \mathscr{N}$ let $\left[Z_{1}, Z_{2}, Z_{3}, \cdots\right]$ denote the sequence of integers appearing in the continued fraction expansion of $Z$.

Lemma 4. There is a Borel measurable function $f$ from $H$ onto $\mathscr{N}$ such that if $Z \in \mathscr{N}$, then $f^{-1}(Z)$ is not a subset of any $F_{\sigma}$ set of Lebesgue measure 0. 
Proof. For each $x \in H$, there is only one sequence of positive integers $\left\{n_{k}\right\}_{k=1}^{\infty}$ such that $x \in \bigcap_{k=1}^{\infty} T_{\left(n_{1}, \cdots, n_{k}\right)}$; let $f(x)$ be the irrational numbers in $\mathscr{N}$ identified with this sequence. It follows from the preceding lemma that $f$ maps $H$ onto $\mathscr{N}$ and if $Z \in \mathscr{N}$, then $f^{-1}(Z)$ is not a subset of an $F_{\sigma}$ set of measure 0 .

For each $k$-tuple $n_{1}, \cdots, n_{k}$, let $J_{\left(n_{1}, \cdots, n_{k}\right)}\left\{Z: Z_{i}=n_{i}, i=1,2, \cdots, k\right\}$. The sets $J_{\left(n_{1}, \cdots, n_{k}\right)}$ form an open base for the usual topology on the space $\mathscr{N}$.

We have

$$
f^{-1}\left(J_{\left(n_{1}, \cdots, n_{k}\right)}\right)=\bigcup_{Z \in \mathscr{N}}\left(\bigcap_{p=1}^{\infty} T_{\left(n_{1}, \cdots, n_{k}, Z_{1}, \cdots, Z_{p}\right)}\right) .
$$

Thus, $f^{-1}\left(J_{\left(n_{1}, \cdots, n_{k}\right)}\right)$ is an analytic set [5, p. 467]. It follows from Lusin's first separation theorem [5, p. 485] that $f$ is Borel measurable (actually, $f^{-1}(U)$ is an $F_{\sigma \delta \sigma}$ set for each open set $U$ ).

We are now in a position to prove

THEOREM 1. There is a Borel measurable function $h$ from the unit interval I onto the Hilbert cube $I^{\omega_{0}}$ such that if $x \in I^{\omega_{0}}$, then $f^{-1}(x)$ is not a subset of an $F_{\sigma}$ set of Lebesgue measure 0.

Proof. Let $f$ be a function as described in Lemma 4. Let $g$ be a con, tinuous function from $\mathscr{N}$ onto the Hilbert cube [5, p. 440]. The composition, $g \circ f$, maps $H$ onto the Hilbert cube and is Borel measurable. Let $\left(g_{1}, g_{2}, g_{3}, \cdots\right)$ be the sequence of the natural projections of $g \circ f$. For each $p, g_{p}$ is a Borel measurable function from $H$ onto the interval $I$ [5, p. 382]. For each $p$, let $\tilde{g}_{p}$ be a Borel measurable extension $g_{p}$ to all of $I$ which maps into $I$. Let $h=\left(\tilde{g}_{1}, \tilde{g}_{2}, \tilde{g}_{3}, \cdots\right)$. The function $h$ has the required properties.

THEOREM 2. There exists a transfinite sequence of "universal functions" $\left\{U_{\alpha}\right\}_{0<\alpha<\omega_{1}}$ such that for each $\alpha, 0<\alpha<\omega_{1}$, we have

(1) $U_{\alpha}$ is a Baire measurable function on $I \times I$ which maps into the unit interval $I$; and

(2) if $f$ is a function in Baire's class a which maps into I, then the set of all $x$ such that $U_{\alpha}(x, y)=f(y)$, for every $y$ in $I$, is not a subset of an $F_{\sigma}$ set of Lebesgue measure zero.

The proof essentially follows the argument in [6, p. 133].

Proof. Let $\left\{s_{n}\right\}_{n=1}^{\infty}$ be a countable dense subset of the positive part of the unit ball of the Banach space $C(I)$.

Let

$$
\begin{aligned}
U_{0}(x, y) & =s_{n}(y), & & \text { if } x=1 / n, \\
& =0, & & \text { otherwise. }
\end{aligned}
$$


It can be easily verified that $U_{0}$ is a Borel measurable function on $I \times I$ and of course it maps into the interval $I$. Let $h=\left(h_{1}, h_{2}, h_{3}, \cdots\right)$ be a function from $I$ onto the Hilbert cube having the properties described in Theorem 1.

For each ordinal $\alpha, 0 \leqq \alpha<\omega_{1}$, let

$$
U_{\alpha+1}(x, y)=\limsup _{p \rightarrow \infty} U_{\alpha}\left(h_{n}(x), y\right)
$$

for each $(x, y) \in I \times I$; also, if $\alpha$ is a limit ordinal, let $\left\{\gamma_{p}\right\}_{p=1}^{\infty}$ be an increasing sequence of ordinals less than $\alpha$ which converges to $\alpha$ and let

$$
U_{\alpha}(x, y)=\limsup _{p \rightarrow \infty} U_{\gamma_{p}}\left(h_{p}(x), y\right) .
$$

It may be proven by transfinite induction that the functions $U_{\alpha}, 0<\alpha<$ $\omega_{1}$, are Borel measurable and map into $I$.

The proof that the functions $U_{\alpha}$ are "universal" and represent each appropriate function in Baire's class $\alpha$ on a "large" set proceeds by transfinite induction.

First, suppose $f$ is in Baire's class 1 and $f$ maps $I$ into $I$. Consequently, there is a sequence $\left(n_{1}, n_{2}, n_{3}, \cdots\right)$ of positive integers such that the sequence $\left\{s_{n_{p}}\right\}_{p=1}^{\infty}$ converges pointwise to $f$ on $I$.

If $x \in h^{-1}\left(1 / n_{1}, 1 / n_{2}, 1 / n_{3}, \cdots\right)$, then

$$
U_{1}(x, y)=\limsup _{p \rightarrow \infty} U_{0}\left(h_{p}(x), y\right)=\limsup _{p \rightarrow \infty} s_{n_{p}}(y)=f(y),
$$

for each $y$ in $I$. Thus, the function $U_{1}$ has the second required property.

Now, suppose $\alpha$ is a limit ordinal, the functions $U_{\gamma}, 0<\gamma<\alpha$, have the required properties and $f$ is a function in Baire's class $\alpha$ which maps $I$ into $I$.

There is a sequence $\left\{f_{p}\right\}_{p=1}^{\infty}$ of functions, converging pointwise to $f$ on $I$ such that for each $p, f_{p}$ is in Baire's class $\gamma_{p}$ and $f_{p}$ maps $I$ into $I$.

For each $p$, let $x_{p}$ be a number in $I$ such that $U_{\gamma_{p}}(x, y)=f_{p}(y)$, for every $y$ in $I$.

If $x \in h^{-1}\left(x_{1}, x_{2}, x_{3}, \cdots\right)$, then $U_{\alpha}(x, y)=f(y)$, for each $y$ in $I$ and $U_{\alpha}$ has the required properties.

A similar argument can be given for the remaining functions $U_{\alpha+1}$.

In order to prove that the Baire order of $\Phi$ is $\omega_{1}$, we will employ a theorem which was published previously by the author:

THEOREM 3 [7]. If $\alpha$ is an ordinal, $0<\alpha<\omega_{1}$, then a function $f$ is in $\Phi_{\alpha}$ if and only if there is a function $g$ in Baire's class $\alpha$ such that the set $D=\{x: f(x) \neq g(x)\}$ is a subset of an $F_{\sigma}$ set of measure zero. 
We will now prove

THEOREM 4. The Baire order of $\Phi$ is $\omega_{1}$.

Proof. Let $\alpha$ be an ordinal, $0<\alpha<\omega_{1}$. Let $U_{\alpha}$ be a universal function having the properties stated in Theorem 2. Let $w(x)=\lim _{n \rightarrow \infty}\left(1-U_{\alpha}(x, x)\right)^{n}$. The function $w$ is a Baire function which maps $I$ into $I$ and there is no $x$ such that $w(x)=U_{\alpha}(x, x)$. Actually, $w$ is the characteristic function of the set of all $x$ such that $U_{\alpha}(x, x)=0$.

Assume that $w \in \Phi_{\alpha}$. By Theorem 3, there is a function $g$ in Baire's class $\propto$ such that the set $D=\{x: w(x) \neq g(x)\}$ is a subset of an $F_{\sigma}$ set $K$ of Lebesgue measure 0 . It is assumed here that $g$ maps into $I$ (this is no restriction). By Theorem 2 , there is some $x \in K^{\prime}$ such that $U_{\alpha}(x, y)=g(y)$ for all $y$ in $I$. In particular, $U_{\alpha}(x, x)=g(x)=w(x)$, since $x \in K^{\prime}$. This contradiction proves the theorem.

Question. If $0<\alpha<\omega_{1}$, is there a $\sigma$-ideal $R_{\alpha}$ of subsets of $I$ of the first category which contains all the sets of Lebesgue measure 0 such that the family $\Phi$ of all functions which are continuous except for a set in this $\sigma$-ideal $R_{\alpha}$ has Baire order $\alpha$ ? See [7], for some relationships between the classes $\Phi_{\alpha}$ and the classical Baire functions of class $\alpha$.

REMARK. As mentioned in the first part of this paper the Baire order of the family of all real-valued functions on $I$ which are continuous except for a first category set is 1 . This fact together with the technique employed in this paper yield the following

THEOREM. There does not exist a Borel measurable function $h$ from the unit interval I onto the Hilbert cube $I^{\omega_{0}}$ having the property that if $x \in I^{\omega_{0}}$, then $f^{-1}(x)$ is not a subset of a first category set.

\section{REFERENCES}

1. H. Lebesgue, Sur les fonctions représentable analytiquement, J. Math. (6) 1 (1905).

2. C. Kuratowski, Sur les fonctions représentables analytiquement et les ensembles de premiere categorie, Fund. Math. 5 (1924), 75-86.

3. L. V. Kantorovitch, Sur les suites des fonctions presque partout continues, Fund. Math. 17 (1930), 25-28.

4. R. D. Mauldin, On Borel measures and Baire's class 3, Proc. Amer. Math. Soc. 39 (1973), 308-312.

5. K. Kuratowski, Topologie, Vol. I, PWN, Warsaw, 1958; English transl., Academic Press, New York; PWN, Warsaw, 1966. MR 19, 873; 36 \#840.

6. I. Natanson, Theory of functions of a real variable, GITTL, Moscow, 1950; English transl. of 1st ed., vol. 1, Ungar, New York, 1955. MR 12, 598; 16, 804.

7. R. D. Mauldin, $\sigma$-ideals and related Baire systems, Fund. Math. 71 (1971), 171177. MR 45 \#2107.

Department of Mathematics, University of Florida, Gainesville, Florida 32601 